\title{
Game of clones: A case of ELANE gene mutation-related neutropenia
}

\author{
N Mashigo, ${ }^{1,2}$ (D) M Esser $^{3}$ \\ ${ }^{1}$ Department of Pathology, Division of Haematology, Faculty of Health Sciences, Stellenbosch University, South Africa \\ ${ }^{2}$ National Health Laboratory Services, South Africa \\ ${ }^{3}$ Department of Pathology, Division of Microbiology, Immunology Unit, Faculty of Health Sciences, Stellenbosch University, South Africa
}

Corresponding author, email: nomusa.mashigo@nhls.ac.za

Mutations in the ELANE gene, which encodes the neutrophil elastase (NE) protein in neutrophils, result in ELANE-related neutropenia. ELANE-related neutropenia encompasses both cyclic neutropenia (CN) and severe congenital neutropenia (SCN), with ELANE mutations seen in a majority of SCN and almost all of $\mathrm{CN}$ patients.

Clinical history in affected individuals typically reveals recurrent episodes of fever, oral ulcerations and bacterial as well as fungal infections, correlating with periodic oscillations or chronically low levels in the absolute neutrophil count (ANC). ELANE-related neutropenia is characterised by severely low neutrophil counts where ANCs may drop as low as zero.

A two-year-old child presented at our hospital with a longstanding history of recurrent bacterial infections and previous admissions at another centre during some of these infectious episodes. Her full blood count demonstrated a markedly low ANC, which was chronic on assessment of her previous results. Decreased granulopoiesis with maturation arrest was seen on her bone marrow and genetic testing for the ELANE gene demonstrated a pathogenic variant of the mutation. Treatment with granulocyte colony-stimulating factor (G-CSF) was initiated.

Keywords: neutrophil elastase, cyclic neutropenia, severe congenital neutropenia, absolute neutrophil count

\section{Introduction}

Neutrophils are short-lived granulocytes that circulate in the blood as quiescent cells and perform their pivotal bactericidal and phagocytic functions outside circulation in the initial defence against microbial invasion. ${ }^{1}$ The level of neutrophils in blood is influenced by various factors, including age, genetic and environmental factors, activity, etc. Neutropenia is a decrease in the neutrophil count below the mean of comparable population and is defined as a decrease in the absolute number of circulating neutrophils to below $1.5 \times 10^{9} / \mathrm{L} .^{2}$ Neutropenia below $0.5 \times 10^{9} / \mathrm{L}$ is considered to be severe, and if it persists for more than three months, it is designated as chronic.

Neutropenia can be classified in various ways, including according to severity (mild, moderate or severe), pattern of variation (cyclic or chronic) or based on whether it is familial or sporadic, amongst a few. With the discovery that mutations in more than 20 genes have been implicated in the causation of neutropenia,
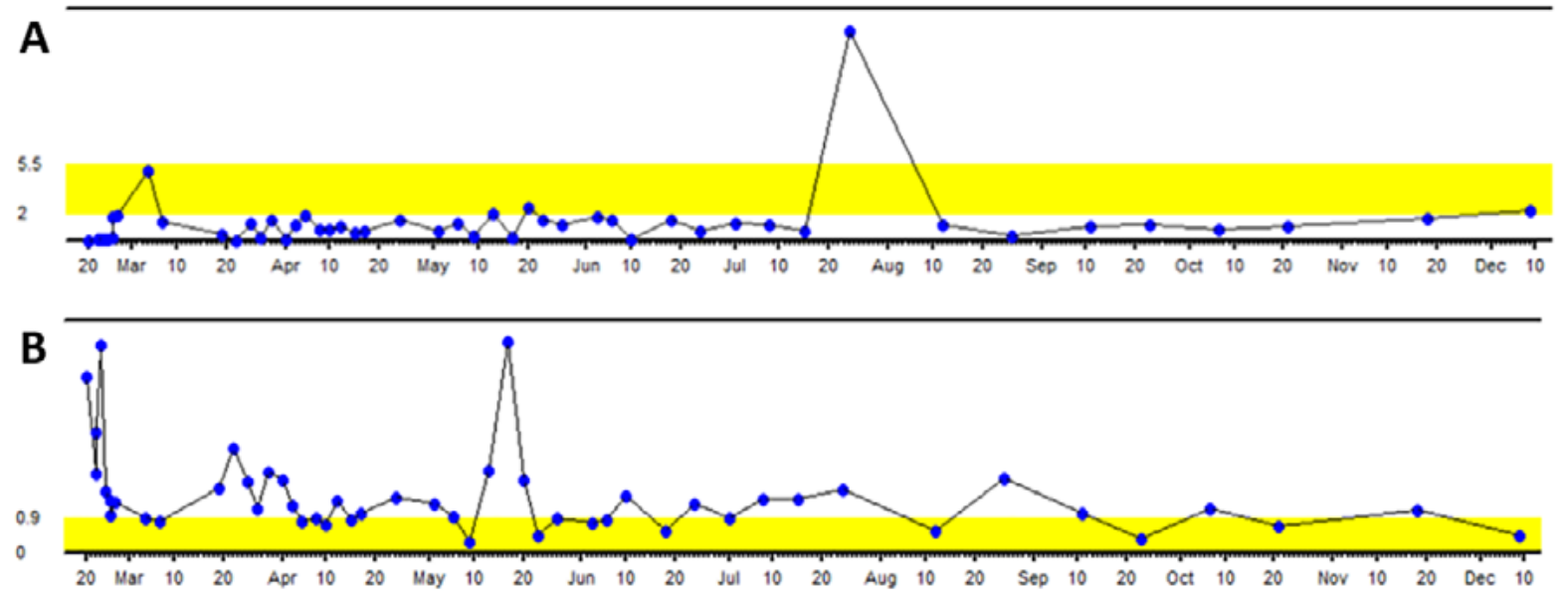

Figure 1: (A) Serial absolute neutrophil counts from the time of diagnosis with occasional nadirs of $0.00 \times 10^{9} / \mathrm{L}$. Improvement noted after a few months of G-CSF therapy with the last count on graph within normal range.

(B) Serial absolute monocyte counts demonstrating inverse trends to the neutrophil pattern. 
it can now additionally be classified according to the specific mutated gene, as is the case with ELANE-related neutropenias.

\section{Case description}

We present a case of a two-year-old child who was seen at our hospital with a well-established history of abscesses and \pm 8 -weekly buccal ulcers. She had had a few previous hospital admissions, one of which was for severe oral mucositis. Her recurrent presentations with bacterial infections started before she was a year old.

Assessment of serial ANCs showed longstanding oscillations in her neutrophil counts, with nadirs of $0.00 \times 10^{9} / \mathrm{L}$ on several occasions. There was persistent relative monocytosis associated with the neutropenia (Figure 1). Bone marrow examination showed reduced and left-shifted granulopoiesis (Figure 2) while cytogenetics demonstrated a normal female karyotype (46 XX).

Genetic testing for the ELANE mutation was done using next generation sequencing and revealed the presence of a pathogenic variant, c.416C $>T$, in the gene. This sequence change replaces proline with leucine at codon 139 of the ELANE protein (p.Pro139Leu). This molecular discovery, together with the clinical findings, laboratory tests and established clinical history, was diagnostic of ELANE-related neutropenia.

The patient was previously treated with a wide variety of antibiotics and was subsequently started on regular granulocyte colony-stimulating factor (G-CSF) injections.

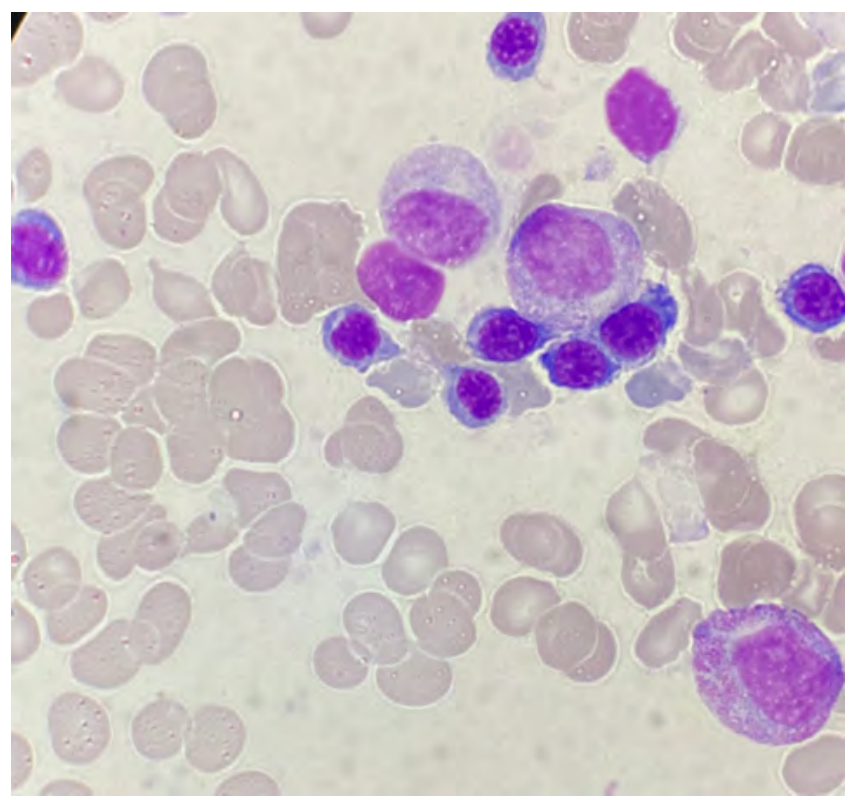

Figure 2: Bone marrow aspirate showing a few promyelocytes, illustrating the maturation arrest in granulopoiesis

\section{Discussion}

Neutrophil elastase (NE) is a serine protease stored in the azurophilic granules of neutrophils along with other bactericidal proteins, and it plays a key role in host defence against bacteria. ${ }^{1,3} \mathrm{NE}$ is encoded by the ELANE gene on chromosome 19 and mutations in this gene have been implicated in both cyclic neutropenia (CN) and severe congenital neutropenia (SCN), with ELANE mutations seen in a majority of $\mathrm{SCN}$ and almost all $\mathrm{CN}$ cases. ${ }^{4,5}$

Cyclic neutropenia is a rare haematological disorder characterised by severe neutropenia typically recurring with a 21-day periodicity and five or more episodes of infections a year as a result of the neutropenia. ${ }^{6}$ It is usually diagnosed within the first year of life and has an estimated frequency of 1:1 000000 in the general population.7 SCN represents a heterogeneous group of haematological disorders also characterised by severe neutropenia and consequent recurrent bacterial infections, with or without an underlying genetic defect. ${ }^{5,8} \mathrm{SCN}$, however, is associated with more severe neutropenia and more serious clinical manifestations than $\mathrm{CN}$ and it confers an additional risk of developing acute myeloid leukaemia (AML) or myelodysplastic syndrome (MDS). $4,5,9$

While classical forms of both disorders may be easily identifiable, a continuum of phenotypes occurs (i.e. variable length of neutropenia in $\mathrm{CN}$ or oscillating neutropenia in $\mathrm{SCN}$ ), because the pathological mechanisms which result in the neutropenia affect both the degree of the nadir and the interval of fluctuations. ${ }^{6,10}$ If an ELANE gene mutation is present, the designation ELANErelated neutropenia or ELANE-associated neutropenia has been used, regardless of the pattern of variation in neutrophils. ${ }^{10}$ It is unclear which factors determine whether a patient with an ELANE mutation will develop an SCN or CN phenotype, however host factors and modifying genes have been proposed to play a role. ${ }^{6,11}$

Several hypotheses have been proposed to explain the pathogenic effects of NE mutations, one of which posits that the neutropenia is attributed to the accelerated apoptosis of neutrophil precursors secondary to mutant neutrophil elastase not being processed and packaged normally in the cells' primary granules. The mutant enzyme impairs survival of myeloid precursors through initiation of the unfolded protein response, leading to programmed cell death and early stage maturation arrest of myelopoiesis (Figure 3)., ${ }^{3,1}$

Horwitz et al. also propose the mislocalisation hypothesis after demonstrating that mutant NE in neutrophils from patients with ELANE mutations was mislocalised within the cell. They postulate that this mislocalisation results in neutropenia as a

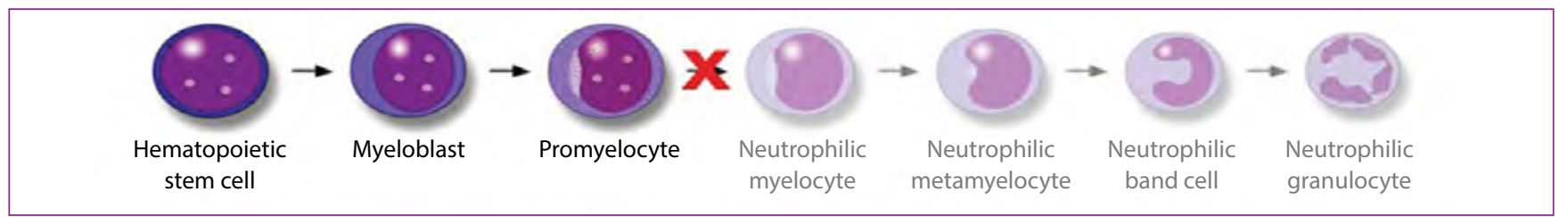

Figure 3: ELANE-related neutropenias are associated with a maturation block at the promyelocyte stage of differentiation. Adapted from Touw ${ }^{11}$ 
result of induced endoplastic reticulum stress which activates the unfolded protein syndrome with resultant apoptosis. ${ }^{4,9}$

The mechanisms which govern the cyclic phenomenon observed in $\mathrm{CN}$ patients has also fascinated clinicians and scientists alike. Numerous theories for these oscillations have been proposed, with a common alignment in most of these models centring around disturbance of a feedback loop mechanism. ${ }^{4}$ This hypothesis proposes that under normal circumstances neutrophils regulate their own production by producing an inhibitor that regulates granulopoiesis, whereby if there's reduction of neutrophils due to infection, for example, then the accompanying low levels of the inhibitor will permit myelopoiesis to proceed. ${ }^{4}$ In this model, there is an inbuilt delay between stimulation and result due to the requisite time for differentiation from early myeloid progenitors to mature neutrophils. When this mechanism is perturbed, there may be excess inhibition of neutrophil production, but only for a short period because synthesis of the inhibitor itself is dependent on neutrophil production, thus myelopoiesis will resume when the inhibitor levels are low due to an exhausted pool of neutrophils. ${ }^{4}$

Diagnosis of ELANE-related neutropenia relies on the clinical presentation and accompanying history, serial measurements of ANC over a period of weeks and demonstration of an ELANE gene mutation by molecular testing. ${ }^{7}$ A bone marrow biopsy is useful in assessing abnormalities in myelopoiesis and may be helpful in demonstrating maturation arrest. Karyotyping using conventional cytogenetics will help in demonstration of any chromosomal lesions which may be associated with specific syndromes if present. Additionally, it is crucial to rule out congenital malformations and investigate for extrahaematopoietic system involvement to exclude other syndromes which are associated with neutropenia (e.g. ShwachmanDiamond disease, etc.). ${ }^{10}$

Management is multi-dimensional and includes treatment of manifestations of the neutropenia (e.g. infections, fever, etc.), prevention of primary manifestations and secondary complications, as well as surveillance for malignant transformation. The introduction of G-CSF was a breakthrough in the treatment of congenital neutropenias and before its widespread use, patients regularly succumbed to life-threatening bacterial infections. G-CSF is given with the aim of achieving and maintaining an ANC $>1 \times 10^{9} / \mathrm{L}$, which is sufficient to mitigate symptoms. ${ }^{9}$ The role of G-CSF in the increased risk for development of AML/MDS in patients with SCN has been long postulated, but it is likely that SCN itself has a contributory effect in the development of the malignancies because patients with $\mathrm{CN}$ who are treated with G-CSF are not at increased risk of developing AML or MDS. ${ }^{5}$

For mutations such as ELANE and HAX1, which are the most frequent, gene corrections using CRISPR/Cas9 gene-editing technology are under investigation as potential new therapeutic avenues. ${ }^{12}$

\section{Conclusion}

This case illustrates the need to consider rare causes in patients presenting with recurrent bacterial or fungal infections and associated neutropenia which may or may not be cyclic. Examination of serial absolute neutrophil counts plus monocyte levels and genetic testing for the ELANE mutation, or any of the more than 20 different germline mutations which have been identified as causative, may help in early definitive diagnosis and prevent long term complications, frequent hospitalisation or risk of mortality. Our patient is currently doing well, reaching and maintaining neutrophil counts within normal range for prolonged periods.

\section{Acknowledgement}

We would like to thank the parents of the patient for allowing us to publish this article on behalf of their daughter.

\section{Conflict of interest}

The authors declare no conflict of interest.

\section{Ethical approval}

Ethical clearance for the study was obtained from the Health Research Ethics Committee of Stellenbosch University. Ethical Clearance number: C20/10/032.

\section{Funding source}

None.

\section{ORCID}

N Mashigo (iD) https://orcid.org/0000-0002-4383-6487

References

1. Kaushansky K, Lichtman MA, Prchal JT, et al. Williams Hematology. 9th ed McGraw-Hill Education; 2016.

2. Bain BJ. Blood cells: a practical guide. 5th ed. West Sussex, UK, John Wiley \& Sons Ltd.; 2015.

3. Köllner I, Sodeik B, Schreek S, et al. Mutations in neutrophil elastase causing congenital neutropenia lead to cytoplasmic protein accumulation and induction of the unfolded protein response. Blood. 2006;108(2):493-500. https://doi. org/10.1182/blood-2005-11-4689.

4. Horwitz MS, Corey SJ, Grimes HL, Tidwell T. ELANE mutations in cyclic and severe congenital neutropenia: genetics and pathophysiology. Hematol Oncol Clin North Am. 2013;27(1):19-41. https://doi.org/10.1016/j.hoc.2012.10.004.

5. Shim YJ, Kim H-J, Suh JS, Lee KS. Novel ELANE gene mutation in severe congenital neutropenia. J Korean Med Sci. 2011;26:1646-49. https://doi. org/10.3346/jkms.2011.26.12.1646.

6. Bellanné-Chantelot $C$, Clauin S, Leblanc T, et al. Mutations in the ELA2 gene correlate with more severe expression of neutropenia: a study of 81 patients from the French Neutropenia Register. Blood. 2004;103(11):4119-25. https://doi. org/10.1182/blood-2003-10-3518.

7. Mansoor MS, Khan MBS. A case of cyclic neutropenia in adults. J Pak Med Assoc 2012;62(3):289-90.

8. Gong R-L, Wu J, Chen T-X. Clinical, laboratory, and molecular characteristics and remission status in children with severe congenital and non-congenita neutropenia. Front Pediatr. 2018;6(305):1-8. https://doi.org/10.3389/ fped.2018.00305.

9. Skokowa J, Dale DC, Touw IP, Zeidler C, Welte K. Severe congenital neutropenias. Nat Rev Dis Primers. 2017;3:17032. https://doi.org/10.1038/nrdp.2017.32.

10. Donadieu J, Beaupain B, Fenneteau O, Bellanné-Chantelot C. Congenital neutropenia in the era of genomics: classification, diagnosis, and natural history. British Journal of Haematology. 2017;179:557-74. https://doi.org/10.1111/ bjh.14887.

11. Touw IP. Game of clones: the genomic evolution of severe congenital neutropenia. Hematology Am Soc Hematol Educ Program. 2015(1):1-7.

12. Welte K, Zeidler C, Skokowa J. Genetic heterogeneity of patients with congenital neutropenias. Educational Updates in Hematology Book: 25th Congress of the European Hematology Association. 2020;4:47-49. https://doi.org/10.1097/ HS9.0000000000000444. 